Pacific

Journal of

Mathematics

TOTAL DETERMINATION OF MATERIAL PARAMETERS FROM ELECTROMAGNETIC BOUNDARY INFORMATION

Mark S. Joshi and Stephen R. McDowall 


\title{
TOTAL DETERMINATION OF MATERIAL PARAMETERS FROM ELECTROMAGNETIC BOUNDARY INFORMATION
}

\author{
Mark S. Joshi And Stephen R. McDowall
}

\begin{abstract}
In this paper we complete the proof that the material parameters can be obtained for a chiral electromagnetic body from the boundary admittance map. We prove that from the admittance map, the parameters are uniquely determined to infinite order at the boundary. This removes the assumption of such knowledge in the result of the second author regarding interior determination for chiral media.
\end{abstract}

\section{Introduction.}

In this paper we complete the proof that the material parameters can be obtained for a chiral electromagnetic body from the boundary admittance map. We achieve this by improving the boundary determination result of [7]. This enables the removal of the assumption in the interior determination result of $[\mathbf{8}]$ in which it is assumed that the material parameters are known to infinite order at the boundary.

The behavior of electromagnetic fields in a body is described by Maxwell's equations. The electric displacement and the magnetic induction of the body are related to the fields by the constituent equations which are defined in terms of a number of material parameters. The parameters typically considered are the conductivity, the electric permittivity and the magnetic permeability. A fourth, often neglected, characteristic of an electromagnetic body is its chirality. Chirality is an asymmetry in the molecular structure: A molecule is chiral if it cannot be superimposed onto its mirror image, and the presence of chirality results in a rotation of electromagnetic fields (see $[5])$.

In [11], Somersalo et. al. presented the boundary admittance map for time-harmonic fields at a fixed frequency for non-chiral bodies, and posed the inverse problem of whether or not the material parameters of a body could be determined from knowledge of this boundary map. In [9] it was shown that this is in fact the case assuming knowledge of the parameters near the boundary. In [8], the second author showed that the admittance map is well defined also in the case of a chiral body, and that knowledge of this map determines the material parameters of the body, including the 
chirality, throughout the body, assuming that the parameters are known to agree to infinite order at the boundary.

It is therefore pertinent to ask whether or not the admittance map in each case determines the material parameters at the boundary of the body. In [7] the second author showed that the admittance map does determine the parameters and their first normal derivatives and it was conjectured that the same should be true for all higher order derivatives. It was infeasible, however, to carry out the computations arrived at in the proof in [7] to prove determination of the higher order derivatives. In the present paper we prove that the admittance map does indeed determine the parameters to infinite order at the boundary in both the chiral and non-chiral cases, thus removing the aforementioned assumptions in the interior determination results. We work exclusively with time-harmonic fields at fixed frequency. In order to pose the problem precisely we shall need the following function spaces: If $\Omega$ is a smoothly bounded open set in $\mathbb{R}^{3}, H^{s}(\Omega)^{k}$ consists of $k$-dimensional vector fields whose components are in the usual $L^{2}$-based Sobolev space $H^{s}$. Let Div denote the surface divergence on the boundary of $\Omega$, and $\nu(x)$ be the outward unit normal vector at $x \in \partial \Omega$, and define the following space of tangential fields:

$$
T H_{\text {Div }}^{\frac{1}{2}}(\partial \Omega)=\left\{F \in H^{\frac{1}{2}}(\partial \Omega)^{3} \mid \nu \cdot F=0, \text { and } \operatorname{Div} F \in H^{\frac{1}{2}}(\partial \Omega)\right\} .
$$

If $F \in T H_{\text {Div }}^{\frac{1}{2}}(\partial \Omega)$, let $(E, H) \in \mathcal{D}^{\prime}(\Omega)^{3} \times \mathcal{D}^{\prime}(\Omega)^{3}$ be the unique solution to Maxwell's equations with boundary condition $\left.\nu \wedge E\right|_{\partial \Omega}=F$. For Maxwell's equations, see Section 1.1 in the non-chiral case, and Section 2.1 in the chiral case. Solvability of the boundary value problem is given in [11] and [8] for non-chiral and chiral bodies respectively. The boundary admittance map $\Lambda: T H_{\text {Div }}^{\frac{1}{2}}(\partial \Omega) \rightarrow T H_{\text {Div }}^{\frac{1}{2}}(\partial \Omega)$ is then defined by

$$
\Lambda F=\left.\nu \wedge H\right|_{\partial \Omega} .
$$

We use $\Lambda$ to denote the non-chiral admittance map, and $\Pi$ to denote the chiral admittance map.

For a non-chiral body, $\varepsilon$ is a complex parameter with real part the electric permittivity and imaginary part $1 / \omega$ times the conductivity of the body, and $\mu$ is the real-valued magnetic permeability of the body. We assume that $|\varepsilon| \geq \varepsilon_{0}>0$ and $\mu \geq \mu_{0}>0$. Suppose that we have two electromagnetic bodies $(\Omega ; \varepsilon, \mu)$ and $\left(\Omega ; \varepsilon^{\prime}, \mu^{\prime}\right)$ with the same boundary $\partial \Omega$. Let $\Lambda$ and $\Lambda^{\prime}$ be the associated admittance maps. By the statement $\Lambda=\Lambda^{\prime}$ we mean the following: For every $F \in T H_{\text {Div }}^{\frac{1}{2}}(\partial \Omega)$, if $(E, H)$ and $\left(E^{\prime}, H^{\prime}\right)$ are the solutions to the boundary value problem above for the bodies $(\Omega ; \varepsilon, \mu)$ and $\left(\Omega ; \varepsilon^{\prime} \mu^{\prime}\right)$ respectively, then

$$
\Lambda F=\left.\nu \wedge H\right|_{\partial \Omega}=\left.\nu \wedge H^{\prime}\right|_{\partial \Omega}=\Lambda^{\prime} F .
$$


We give the analogous meaning to $\Pi=\Pi^{\prime}$.

We may now state the results. In Section 1 we prove:

Theorem A. Let $(\Omega ; \varepsilon, \mu)$ and $\left(\Omega ; \varepsilon^{\prime}, \mu^{\prime}\right)$ be bounded electromagnetic bodies in $\mathbb{R}^{3}$ with electromagnetic parameters $(\varepsilon, \mu),\left(\varepsilon^{\prime}, \mu^{\prime}\right) \in C^{\infty}\left(\mathbb{R}^{3}\right)$, and with smooth boundary $\partial \Omega$. Let $\Lambda$ and $\Lambda^{\prime}$ be the associated admittance maps, and assume that $\Lambda=\Lambda^{\prime}$. Then on $\partial \Omega$

$$
\varepsilon=\varepsilon^{\prime}, \quad \text { and } \quad \mu=\mu^{\prime}
$$

and the same is true of all derivatives at the boundary.

In Section 2 we handle the case of a chiral body. The method of proof is essentially the same as in the non-chiral case, however the underlying ideas become somewhat obscured by the more complicated setting, and for this reason we have presented the two proofs separately, the first illustrating the ideas more accessibly. The reason for the difference between the proofs is that the non-chiral Maxwell's equations decouple in a way that the chiral equations do not. The parameters describing the electromagnetic properties of a chiral body used here are (as in [8]) a change of variables from the ones in the Born-Federov formulation (see [5]). The parameters $\varepsilon$ and $\mu$ satisfy the same conditions as for the non-chiral case above, and $\beta$ describes the chirality, and is a purely imaginary smooth function. We assume that $\varepsilon \mu+\beta^{2} \neq 0$ which amounts to assuming that the fields never become parallel.

The result of Section 2 is:

Theorem B. Let $(\Omega ; \varepsilon, \mu, \beta)$ and $\left(\Omega ; \varepsilon^{\prime}, \mu^{\prime}, \beta^{\prime}\right)$ be two bounded electromagnetic bodies in $\mathbb{R}^{3}$ with chirality described by $\beta$ and $\beta^{\prime}$ respectively. Suppose that $(\varepsilon, \mu, \beta),\left(\varepsilon^{\prime}, \mu^{\prime}, \beta^{\prime}\right)$ are in $C^{\infty}\left(\mathbb{R}^{3}\right)$ and $\partial \Omega$ is smooth. If the associated admittance maps are equal, $\Pi=\Pi^{\prime}$, then on $\partial \Omega$

$$
\varepsilon=\varepsilon^{\prime}, \quad \mu=\mu^{\prime}, \quad \text { and } \quad \beta=\beta^{\prime}
$$

and the same is true of all derivatives at the boundary.

It was shown in [7], although not stated explicitly, that the admittance maps are pseudo-differential operators of order one. Our approach is an inductive one. We will assume that the Taylor series of the parameters are known to agree to some order at the boundary, and then compute the principal symbol of the difference of the two associated admittance maps. We show that this principal symbol then determines the next term in the Taylor series of the parameters. This approach has already been exploited in various inverse scattering problems, $[\mathbf{1}],[2],[3]$ and $[4]$. Computing the principal symbol turns out to be computationally much simpler than trying to compute the lower order terms of the symbol of a single admittance map as we are able to work invariantly and solely with principal symbols. This is facilitated by the study of families of classical pseudo-differential operators $P_{t} \in \Psi D O_{c l}^{m}$ parameterized by normal distance from the boundary. These 
have the property that the total symbol is of lower order when restricted to the boundary. We are then able to define an invariant vector of principal symbols for this family by taking the principal symbols of $\left.\frac{1}{l !} \frac{d^{l}}{d t} P_{t}\right|_{t=0}$. We study and utilize the properties of this vector under composition with normal differentiation at the boundary and with pseudo-differential operators.

In [8] it was shown that for a chiral body the admittance map uniquely determines the material parameters throughout the body under the assumption that they are known to infinite order at the boundary. We thus have the following corollary:

Corollary C. With $(\Omega ; \varepsilon, \mu, \beta)$ and $\left(\Omega ; \varepsilon^{\prime}, \mu^{\prime}, \beta^{\prime}\right)$ as in Theorem $\mathrm{B}$, if $\Pi=$ $\Pi^{\prime}$ then

$$
\varepsilon=\varepsilon^{\prime}, \quad \mu=\mu^{\prime}, \quad \text { and } \quad \beta=\beta^{\prime}
$$

throughout $\Omega$.

We thus conclude that, in principle, the material parameters of a chiral electromagnetic body are recoverable from information obtained only at the boundary of the body, namely knowledge of the boundary admittance map.

\section{The Non-Chiral Case.}

1.1. The equations. We assume that we are working in a compact convex body, $\Omega$, in $\mathbb{R}^{3}$ and that we have picked geodesic normal coordinates local to a point on the boundary so that $\partial \Omega$ is $x_{3}=0$ and the Euclidean metric becomes

$$
d x_{3}^{2}+\sum_{i, j<3} g_{i j}(x) d x_{i} d x_{j} .
$$

For the moment, we make no constraints on $g_{i j}$ but shall do so later.

As we have a metric, there is a natural pairing and therefore an isomorphism between one-forms and vector fields - we shall work exclusively with one-forms. For time-harmonic fields at fixed frequency $\omega$, Maxwell's equations then take the form

$$
\begin{aligned}
& * d H=-i \omega \varepsilon E \\
& * d E=i \omega \mu H
\end{aligned}
$$

and

$$
\delta(\varepsilon E)=0, \quad \delta(\mu H)=0 .
$$

Here $*$ is the Hodge star operator, $d$ is exterior differentiation and $\delta=* d *$ is its adjoint. We have

$$
* d\left(\frac{1}{\mu} * d E\right)=\omega^{2} \varepsilon E
$$


and thus

$$
\mu * d\left(\frac{1}{\mu}\right) \wedge * d E+* d * d E=\omega^{2} \mu \varepsilon E
$$

Or

$$
-* d(\log \mu) \wedge * d E+* d * d E=\omega^{2} \mu \varepsilon E .
$$

Now on one-forms on a three-manifold, $\Delta=-* d * d+d * d *$ so

$$
-\Delta E+d * d * E-*(d \log \mu \wedge * d E)-\omega^{2} \mu \varepsilon E=0 .
$$

We also know

$$
\delta(E)=-E \cdot d \log \varepsilon .
$$

(Here we are taking the inner product on one-forms induced by the metric - this is an invariant statement and holds in the flat coordinates.) So

$$
-\Delta E-d(E \cdot d \log \varepsilon)-*(d \log \mu \wedge * d E)-\omega^{2} \mu \varepsilon E=0 .
$$

This is of the form

$$
\mathcal{M} E=\left[D_{x_{3}}^{2} I-\Delta_{x_{3}}^{\prime} I-M\left(x, D_{x^{\prime}}\right)-i P(x) D_{x_{3}}-R(x)\right] E=0,
$$

where as in [7] $M$ consists of all terms involving first order differentiation in $x_{1}$ and $x_{2}, P$ is the coefficient matrix of $\partial / \partial_{x_{3}}, R$ consists of all zero order terms and $\Delta_{x_{3}}^{\prime}$ is the Laplacian in $x_{1}, x_{2}$ for the value of $x_{3}$.

\subsection{Factorization.}

Proposition 1.1. There exists $B\left(x, D_{x^{\prime}}\right) \in \Psi D O^{1}$ with principal symbol $-\left|\xi^{\prime}\right|_{x}$ depending smoothly on $x_{3}$ such that

$$
\mathcal{M}=\left(D_{x_{3}} I-i P(x)-i B\right)\left(D_{x_{3}} I+i B\right)
$$

up to smoothing and $B$ is unique up to smoothing.

Proof. As in [7]

$$
\Delta^{\prime}+i\left[D_{x_{3}}, B\right]+M+P B+B^{2}+R=0 .
$$

The principal symbol of $B$ is therefore $\pm\left|\xi^{\prime}\right|_{x}$; we take $-\left|\xi^{\prime}\right|_{x}$. Now suppose we have chosen $B_{j} \in \Psi D O^{1-j}$ such that

$$
G_{N}=B_{0}+B_{1}+\cdots+B_{N}
$$

satisfies

$$
\Delta^{\prime}+i\left[D_{x_{3}}, G_{N}\right]+M+P G_{N}+G_{N}^{2}+R=E_{N} \in \Psi D O^{1-N} .
$$

Then we must have

$$
i\left[D_{x_{3}}, B_{N+1}\right]+P B_{N+1}+B_{N+1}^{2}+G_{N} B_{N+1}+B_{N+1} G_{N}-E_{N} \in \Psi D O^{-N} .
$$

The principal symbol of this is just

$$
-2\left|\xi^{\prime}\right|_{x} \sigma_{-N}\left(B_{N+1}\right)-\sigma_{1-N}\left(E_{N}\right) .
$$


So picking the symbol of $B_{N+1}$ appropriately this is zero away from $\xi^{\prime}=0$ and we can construct all the $B_{N}$; we let $B$ be the asymptotic sum. By construction, $B$ is unique modulo a smoothing operator.

1.3. A class of pseudo-differential operators. Let $Y$ be a smooth manifold. We study families of operators on $Y$ smoothly varying with a parameter $t$. (We will take $Y$ to be $\partial \Omega$ and $t$ the normal distance.)

Definition 1.2. We shall say $P \in \Psi D O^{m, p}\left(Y ; \mathbb{R}_{+}\right)$if it is a family of pseudo-differential operators of order $m$ on $Y$, varying smoothly up to $t=0$, and such that

$$
P=\sum_{j=0}^{p} t^{p-j} P_{j}
$$

with $P_{j}$ a smooth family of operators on $Y$ of order $m-j$. We will allow operators on bundles also.

Definition 1.3. If $P \in \Psi D O^{m, p}\left(Y ; \mathbb{R}_{+}\right)$then the symbol of $P$ at $t=0$ is the vector

$$
\left(\sigma_{m-j}\left(P_{j}\right)\right)_{j=0}^{p}
$$

evaluated at $t=0$. This makes invariant sense as a vector of functions on the cotangent bundle of $Y$.

The following is immediate.

Proposition 1.4. If $B$ is a smooth family of operators on $Y$ of order $k$ and $P \in \Psi D O^{m, p}\left(Y ; \mathbb{R}_{+}\right)$then $B P, P B \in \Psi D O^{m+k, p}\left(Y ; \mathbb{R}_{+}\right)$and the symbols of $P B, B P$ at $t=0$ are just the product of the symbols. We also have that

$$
\left[D_{t}, P\right] \in \Psi D O^{m, p-1}
$$

and that the principal symbol at the boundary is $-i\left((p-j) \sigma_{m-j}\left(P_{j}\right)\right)_{j=0}^{p-1}$.

1.4. The symbol of the difference. Now suppose we have two different sets of parameters, $(\varepsilon, \mu)$ and $\left(\varepsilon^{\prime}, \mu^{\prime}\right)$, with associated admittance maps $\Lambda$ and $\Lambda^{\prime}$; let $B$ and $B^{\prime}$ be the associated factorization operators from Proposition 1.1. We shall consistently use ' to denote operators associated to $\varepsilon^{\prime}, \mu^{\prime}$.

Suppose that $\Lambda=\Lambda^{\prime}$; from [7] we know that on $\partial \Omega \varepsilon=\varepsilon^{\prime}, \mu=\mu^{\prime}$ and the same is true of the first normal derivatives. We shall prove inductively that if $\varepsilon$ and $\varepsilon^{\prime}$ are known to agree to order $l \geq 2$ at the boundary, and similarly for $\mu$ and $\mu^{\prime}$, then the fact that $\Lambda=\Lambda^{\prime}$ implies that the parameters agree to order $l+1$. To this end we write

$$
\varepsilon / \varepsilon^{\prime}=1+x_{3}^{l} e_{\varepsilon}, \quad \mu / \mu^{\prime}=1+x_{3}^{l} e_{\mu},
$$

with $e_{\varepsilon}, e_{\mu}$ smooth up to the boundary. We will calculate $e_{\varepsilon}$ and $e_{\mu}$ at the boundary from the principal symbol of the difference $\Lambda^{\prime}-\Lambda$ which we write 
in terms of the difference $B^{\prime}-B$. We fix a point $p$ on the boundary and work in coordinates which are geodesic normal coordinates at $p$ in the boundary and extended normally off the boundary.

Proposition 1.5. Suppose $(\varepsilon, \mu)$ and $\left(\varepsilon^{\prime}, \mu^{\prime}\right)$ are equal to order $l \geq 2$ at the boundary. Writing $B^{\prime}=B+F$ we have $F \in \Psi D O^{0, l-1}$, and if $\left(f_{j}\right)_{j=0,-(l-1)}$ is the principal symbol of $F$ at the boundary, then in our chosen local boundary normal coordinates

$$
f_{-(l-1)}=\frac{l !}{2^{l}\left|\xi^{\prime}\right|_{x}^{l}}\left[e_{\varepsilon}\left(\begin{array}{ccc}
0 & 0 & i \xi_{1} \\
0 & 0 & i \xi_{2} \\
0 & 0 & \left|\xi^{\prime}\right|_{x}
\end{array}\right)+e_{\mu}\left(\begin{array}{ccc}
\left|\xi^{\prime}\right|_{x} & 0 & i \xi_{1} \\
0 & \left|\xi^{\prime}\right|_{x} & i \xi_{2} \\
0 & 0 & 0
\end{array}\right)\right]+r^{\prime}
$$

where $r^{\prime}$ vanishes to second order at $x_{1}, x_{2}=0$.

Proof. With $B^{\prime}=B+F$ we have

$$
\begin{gathered}
\Delta^{\prime}+i\left[D_{x_{3}}, B\right]+M+P B+B^{2}+R=0, \quad \text { and } \\
\Delta^{\prime}+i\left[D_{x_{3}}, B+F\right]+M^{\prime}+P^{\prime}(B+F)+(B+F)^{2}+R^{\prime}=0 .
\end{gathered}
$$

Subtracting yields,

$$
i\left[D_{x_{3}}, F\right]+P^{\prime} F+F^{2}+F B+B F=\left(M^{\prime}-M\right)+\left(R^{\prime}-R\right)+\left(P^{\prime}-P\right) B .
$$

So if we can construct $F$ satisfying (1.4), then $B^{\prime}=B+F$ as we know $B^{\prime}$ is unique (modulo smoothing).

The terms on the right hand side of (1.4) result from $-\omega^{2} \varepsilon \mu E$, $d(E \cdot d \log \varepsilon)$ and $*(d \log \mu \wedge * d E)$ (see Section 1.1). Specifically, the terms involving differentiation in the tangential variables make up $\left(M^{\prime}-M\right)$, and those involving no differentiation make up $\left(R^{\prime}-R\right)$; the coefficients of differentiation with respect to the normal variable $x_{3}$ are what comprise $\left(P^{\prime}-P\right)$, which then multiplies the first order pseudo-differential operator $B$. We compute the contribution of these terms to the right hand side of (1.4) modulo $\Psi D O^{1, l}$. Now

$$
\omega^{2} \varepsilon \mu-\omega^{2} \varepsilon^{\prime} \mu^{\prime}=\omega^{2} \varepsilon^{\prime} \mu^{\prime}\left(x_{3}^{l}\left(e_{\varepsilon}+e_{\mu}\right)+x_{3}^{2 l} e_{\varepsilon} e_{\mu}\right) \in \Psi D O^{0, l} .
$$

We also have,

$$
\begin{aligned}
d\left(E \cdot d \log \varepsilon^{\prime}\right)-d(E \cdot d \log \varepsilon) & =-d\left(E \cdot d \log \left(\frac{\varepsilon}{\varepsilon^{\prime}}\right)\right) \\
& =-d\left(E \cdot d \log \left(1+x_{3}^{l} e_{\varepsilon}\right)\right)
\end{aligned}
$$

and

$$
d\left[\log \left(1+x_{3}^{l} e_{\varepsilon}\right)\right]=\frac{l x_{3}^{l-1} e_{\varepsilon} d x_{3}}{1+x_{3}^{l} e_{\varepsilon}}+\frac{x_{3}^{l} d e_{\varepsilon}}{1+x_{3}^{l} e_{\varepsilon}} .
$$


Thus

$$
E \cdot d \log \left(1+x_{3}^{l} e_{\varepsilon}\right)=\frac{l x_{3}^{l-1} e_{\varepsilon} E_{3}}{1+x_{3}^{l} e_{\varepsilon}}+\frac{x_{3}^{l} E \cdot d e_{\varepsilon}}{1+x_{3}^{l} e_{\varepsilon}}
$$

and so

$$
\begin{aligned}
d\left[E \cdot d \log \left(1+x_{3}^{l} e_{\varepsilon}\right)\right]= & \frac{l(l-1) x_{3}^{l-2} e_{\varepsilon} E_{3}}{1+x_{3}^{l} e_{\varepsilon}} d x_{3}+\frac{l x_{3}^{l-1} E_{3}}{1+x_{3}^{l} e_{\varepsilon}} d e_{\varepsilon} \\
& +\frac{l x_{3}^{l-1} e_{\varepsilon}}{1+x_{3}^{l} e_{\varepsilon}} d E_{3}+\frac{l x_{3}^{l-1}\left(E \cdot d e_{\varepsilon}-x_{3}^{l-1} e_{\varepsilon}^{2} E_{3}\right)}{\left(1+x_{3}^{l} e_{\varepsilon}\right)^{2}} d x_{3} \\
& -\frac{x_{3}^{2 l}\left(l x_{3}^{-1} e_{\varepsilon} E_{3}+E \cdot d e_{\varepsilon}\right)}{\left(1+x_{3}^{l} e_{\varepsilon}\right)^{2}} d e_{\varepsilon}+\frac{x_{3}^{l}}{1+x_{3}^{l} e_{\varepsilon}} d\left(E \cdot d e_{\varepsilon}\right) \\
= & T_{1}+T_{2}+T_{3}+T_{4}+T_{5}+T_{6}, \quad \text { say. }
\end{aligned}
$$

We compute the contribution of $d\left(E \cdot d \log \left(1+x_{3}^{l} e_{\varepsilon}\right)\right)$ to the right hand side of (1.4) modulo $\Psi D O^{1, l}$; this means we can ignore operators not involving $D_{x_{3}}$ if they are in $\Psi D O^{1, l}$, and so we can drop the terms $T_{2}, T_{4}, T_{5}$. The coefficients of operators in $D_{x_{3}}$ are what make up $P^{\prime}-P$ which, in the right hand side of (1.4), is multiplied by $B$. The resulting operator $\left(P^{\prime}-P\right) B$ is in $\Psi D O^{1, l}$ if the coefficients vanish to order $l$. Thus we may drop $T_{6}$.

We are left with

$$
d\left(E \cdot d \log \left(1+x_{3}^{l} e_{\varepsilon}\right)\right)=\frac{1}{1+x_{3}^{l} e_{\varepsilon}}\left(l(l-1) x_{3}^{l-2} e_{\varepsilon} E_{3} d x_{3}+l x_{3}^{l-1} e_{\varepsilon} d E_{3}+T\right)
$$

where the contribution of $T$ in (1.1) results in operators in $\Psi D O^{1, l}$. The $d E_{3}$ will have two parts - the coefficient of $D_{x_{3}}$ is absorbed into $P^{\prime}-P$ and then multiplied by $B$. Thus modulo $\Psi D O^{1, l}$ the symbolic contribution of $-d\left(E \cdot d \log \left(1+x_{3}^{l} e_{\varepsilon}\right)\right)$ to the right hand side is

$$
\frac{-l(l-1) x_{3}^{l-2} e_{\varepsilon}}{1+x_{3}^{l} e_{\varepsilon}}\left(\begin{array}{ccc}
0 & 0 & 0 \\
0 & 0 & 0 \\
0 & 0 & 1
\end{array}\right)-\frac{l x_{3}^{l-1} e_{\varepsilon}}{1+x_{3}^{l} e_{\varepsilon}}\left(\begin{array}{ccc}
0 & 0 & i \xi_{1} \\
0 & 0 & i \xi_{2} \\
0 & 0 & -\left|\xi^{\prime}\right|_{x}
\end{array}\right) .
$$

This leaves the term $*(d \log (\mu) \wedge * d E)$. When we take the difference we get $-*\left(d \log \left(1+x_{3}^{l} e_{\mu}\right) \wedge * d E\right)$. The only term from this which does not result in something in the right hand side of (1.1) absorbable into $\Psi D O^{1, l}$ is

$$
\frac{-l x_{3}^{l-1} e_{\mu}}{1+x_{3}^{l} e_{\mu}} *\left(d x_{3} \wedge * d E\right) .
$$

Now the Hodge star operator in these coordinates is equal to the flat star operator, $*_{f}$, in these coordinates plus an error. We now assume that we have chosen coordinates normal about some point in the boundary and then 
extended normally away from the boundary. Then

$$
*=\left(I+x_{3} D+\sum_{i, j<3} x_{i} x_{j} * D_{i j}\right) *_{f},
$$

with $D, D_{i j}$ smooth homomorphisms of the form bundles. The $x_{3} D$ will give us an element of $\Psi D O^{1, l}$ which is therefore ignorable. We first compute in flat coordinates the value of $*_{f}\left(d x_{3} \wedge *_{f} d E\right)$; this is equal to

$$
-\frac{\partial E_{1}}{\partial x_{3}} d x_{1}+\frac{\partial E_{3}}{\partial x_{1}} d x_{1}-\frac{\partial E_{2}}{\partial x_{3}} d x_{2}+\frac{\partial E_{3}}{\partial x_{2}} d x_{2} .
$$

As before the differentiation in $x_{3}$ becomes part of $P^{\prime}-P$ and we therefore have to drop the $\xi_{3}$ in the symbol and replace it by the principal symbol of $B$ which is $-\left|\xi^{\prime}\right|_{x}$, so we conclude that the contribution to the forcing on the right hand side is

$$
\frac{-l x_{3}^{l-1} e_{\mu}}{1+x_{3}^{l} e_{\mu}}\left(\left(\begin{array}{ccc}
0 & 0 & i \xi_{1} \\
0 & 0 & i \xi_{2} \\
0 & 0 & 0
\end{array}\right)+\left|\xi^{\prime}\right|_{x}\left(\begin{array}{ccc}
1 & 0 & 0 \\
0 & 1 & 0 \\
0 & 0 & 0
\end{array}\right)\right)+\sigma\left(G_{1}\right)+\sigma\left(G_{2}\right) .
$$

Here $G_{1} \in \Psi D O^{1, l}$ and $G_{2}=\sum_{j, j<3} x_{i} x_{j} G_{i j}$ with $G_{i j} \in \Psi D O^{1, l-1}$.

So we have that $i\left[D_{x_{3}}, F\right]+P^{\prime} F+F^{2}+F B+B F=G$ with $G \in \Psi D O^{1, l-1}$ from (1.4). We first show that this means $F \in \Psi D O^{0, l-1}$ and then compute the symbol of $F$ at our chosen point.

We know $F$ is a pseudo-differential operator of order zero as $B, B^{\prime}$ have the same principal symbol. The principal symbol of the left hand side is $-2\left|\xi^{\prime}\right|_{x} \sigma_{0}(F)$ and so we pick $F_{0}$ to have principal symbol $-1 /\left(2\left|\xi^{\prime}\right|_{x}\right) \sigma_{1}(G)$ which vanishes to order $l-1$ at $x_{3}=0$, so we can certainly take $F_{0} \in$ $\Psi D O^{0, l-1}$. Putting $F_{1}=F-F_{0}$ we then obtain a similar equation but with right hand side in $\Psi D O^{0, l-2}$ and thus solve to get $F_{1} \in \Psi D O^{-1, l-2}$. Repeating we get $F_{0}+F_{1}+\cdots+F_{l-1} \in \Psi D O^{0, l-1}$ solving up to an error in $\Psi D O^{-l}$. This can then be removed in the same way $B$ was originally constructed so we conclude that $F=B-B^{\prime}$ can be constructed to be in $\Psi D O^{0, l-1}$ and therefore by uniqueness is actually in $\Psi D O^{0, l-1}$.

Now recall that the principal symbol of $F$ at $x_{3}=0$ is a well-defined object and is a vector of matrices. Let $\left(f_{j}\right)_{j=0,-(l-1)}$ be this vector. Then $F B+B F$ has symbol $\left(-2\left|\xi^{\prime}\right|_{x} f_{j}\right)$ and $i\left[D_{x_{3}}, F\right] \in \Psi D O^{0, l-2}$ has symbol $\left((l-1+j) f_{j}\right)_{j=0}^{-(l-2)}$. So from $(1.4)$, we obtain that

$$
\begin{aligned}
& -2\left|\xi^{\prime}\right|_{x} f_{0}= \\
& -l e_{\mu}\left(\left(\begin{array}{ccc}
0 & 0 & i \xi_{1} \\
0 & 0 & i \xi_{2} \\
0 & 0 & 0
\end{array}\right)+\left|\xi^{\prime}\right|_{x}\left(\begin{array}{ccc}
1 & 0 & 0 \\
0 & 1 & 0 \\
0 & 0 & 0
\end{array}\right)\right)-l e_{\varepsilon}\left(\begin{array}{ccc}
0 & 0 & i \xi_{1} \\
0 & 0 & i \xi_{2} \\
0 & 0 & -\left|\xi^{\prime}\right|_{x}
\end{array}\right)+r
\end{aligned}
$$


where $r$ vanishes to second order at $x_{1}, x_{2}=0$. We also have

$$
-2\left|\xi^{\prime}\right|_{x} f_{-1}+(l-1) f_{0}=-l(l-1) e_{\varepsilon}\left(\begin{array}{ccc}
0 & 0 & 0 \\
0 & 0 & 0 \\
0 & 0 & 1
\end{array}\right)
$$

and

$$
-2\left|\xi^{\prime}\right|_{x} f_{-j}+(l-j) f_{1-j}=0 .
$$

We can thus iteratively determine $f_{-(l-1)}$, the principal symbol of $F$ restricted to $x_{3}=0$, which is what we want to know. Explicitly,

$$
f_{-(l-1)}=\frac{l !}{2^{l}\left|\xi^{\prime}\right|_{x}^{l}}\left[e_{\varepsilon}\left(\begin{array}{ccc}
0 & 0 & i \xi_{1} \\
0 & 0 & i \xi_{2} \\
0 & 0 & \left|\xi^{\prime}\right|_{x}
\end{array}\right)+e_{\mu}\left(\begin{array}{ccc}
\left|\xi^{\prime}\right|_{x} & 0 & i \xi_{1} \\
0 & \left|\xi^{\prime}\right|_{x} & i \xi_{2} \\
0 & 0 & 0
\end{array}\right)\right]+r^{\prime}
$$

where $r^{\prime}$ vanishes to second order at $x_{1}, x_{2}=0$.

1.5. Recovering the coefficients. In [7] it was shown that the parameters and their first derivatives are determined on the boundary from the admittance map. As in the previous section we assume that $(\varepsilon, \mu)$ and $\left(\varepsilon^{\prime}, \mu^{\prime}\right)$ are known to agree to order $l \geq 2$ on the boundary. In this section we show that the difference of the admittance maps, $\Lambda^{\prime}-\Lambda$, determines $e_{\varepsilon}$ and $e_{\mu}$ (see (1.2)). We fix a point $p$ in the boundary of the domain, take normal coordinates in the boundary about it and then extend these off the boundary normally.

Before proceeding to the proof, we present a useful lemma.

Lemma 1.6. Suppose that $P_{1}$ and $P_{2}$ are pseudo-differential operators of the form

$$
P_{j}=A_{j}+f\left(x^{\prime}\right) R_{j}
$$

where $A_{j}, R_{j}$ are $m^{\text {th }}$-order pseudo-differential operators, $j=1,2$, and $f(p)=0$. Then we can recover the principal symbol of $A_{1}-A_{2}$ at $p$ (even if it is of lower order than $\left.P_{1}-P_{2}\right)$.

This follows from the fact that in any coordinate system the total left symbol of $f\left(x^{\prime}\right)\left(R_{1}-R_{2}\right)$ will vanish at $p$. The usefulness lies in our ability to discard terms in otherwise complicated computations.

From the definition of $\Lambda$ (0.1), Maxwell's equations (1.1), and the expansion for the Hodge star operator (1.5), the admittance map at the point $p$ is

$$
\left.\left.\left(\begin{array}{c}
E_{2} \\
-E_{1}
\end{array}\right)\right|_{x_{3}=0} \mapsto \frac{1}{i \omega \mu}\left(\begin{array}{c}
\partial_{3} E_{1}-\partial_{1} E_{3} \\
\partial_{3} E_{2}-\partial_{2} E_{3}
\end{array}\right)\right|_{x_{3}=0}
$$

plus terms which have coefficients vanishing at $x_{1}=x_{2}=0$; we shall be considering the difference $\Lambda^{\prime}-\Lambda$ and so from the above lemma, the symbol of $\Lambda^{\prime}-\Lambda$ at $p$ does not involve these terms. 
We wish to express the right hand side in terms of $E_{1}$ and $E_{2}$ and operators solely on the boundary. We know that $\delta(\varepsilon E)=0$ and hence that $d *(\varepsilon E)=0$. As above,

$$
*=\left(\mathrm{Id}+x_{3} D+\sum_{i, j} x_{i} x_{j} D_{i j}\right) *_{f}
$$

with $D, D_{i j}$ variable matrices (all sums are taken over $i, j \leq 2$ ). So we have that

$$
\begin{aligned}
& d \varepsilon \wedge\left(\left(\operatorname{Id}+x_{3} D+\sum_{i j} x_{i} x_{j} D_{i j}\right) *_{f} E\right) \\
& +\varepsilon d\left(\left(\operatorname{Id}+x_{3} D+\sum_{i j} x_{i} x_{j} D_{i j}\right) *_{f} E\right)=0 .
\end{aligned}
$$

Evaluating at $x_{3}=0$ this becomes,

$$
\begin{aligned}
d \varepsilon \wedge\left(*_{f} E\right)+\sum_{i j} & x_{i} x_{j} d \varepsilon \wedge\left(D_{i j} *_{f} E\right)+\varepsilon\left(d *_{f} E\right) \\
& +\varepsilon\left(d x_{3} \wedge\left(D *_{f} E\right)\right)+\varepsilon \sum_{i j} d\left(x_{i} x_{j} D_{i j} *_{f} E\right)=0 .
\end{aligned}
$$

Proposition 1.7. If $(U, x)$ is a local geodesic normal coordinate patch to the boundary of $\Omega$ near $p$, then

$$
\frac{\partial E_{j}}{\partial x_{3}}=\sum_{l=1}^{3} B_{j l} E_{l}, \quad j=1,2,3
$$

where $B_{j l}$ are the components of the matrix of operators $B$.

The proof of this is as in [7] Proposition 2, and [6] Proposition 1.2. So dividing by $\varepsilon$ and rearranging, we conclude that,

$$
\begin{gathered}
\left(\operatorname{Id}+\sum_{i, j} x_{i} x_{j} D_{i j}\right)\left(B_{33}+\partial_{3} \log \varepsilon\right) E_{3}+\left(D+\sum_{i, j} x_{i} x_{j} \partial_{3} D_{i j}\right) E_{3} \\
=-\left(\operatorname{Id}+\sum_{i, j} x_{i} x_{j} D_{i j}\right)\left[\left(\partial_{1} \log \varepsilon\right) E_{1}+\left(\partial_{2} \log \varepsilon\right) E_{2}\right. \\
\left.+\partial_{1} E_{1}+\partial_{2} E_{2}+B_{31} E_{1}+B_{32} E_{2}\right] \\
-\partial_{1}\left(\sum_{i, j} x_{i} x_{j} D_{i j}\right) E_{1}-\partial_{2}\left(\sum_{i, j} x_{i} x_{j} D_{i j}\right) E_{2}=T, \text { say. }
\end{gathered}
$$


Let $J$ be the coefficient of $E_{3}$, and let $K$ be a parametrix for $J$ which is certainly elliptic at $x_{1}, x_{2}=0$. We also have that the principal symbol of $K$ is just $-\left|\xi^{\prime}\right|_{x}^{-1} \mathrm{Id}$.

So $E_{3}=K T$ and

$$
\partial_{3} E_{1}-\partial_{1} E_{3}=B_{11} E_{1}+B_{12} E_{2}+\left(B_{13}-\partial_{1}\right) K T .
$$

Extracting the components acting on $E_{2}$, we have

$$
\begin{aligned}
& i \omega \mu \Lambda_{11} \\
& =B_{12}-\left(B_{13}-\partial_{1}\right) K\left[\left(\operatorname{Id}+\sum_{i, j} x_{i} x_{j} D_{i j}\right)\left(\partial_{2} \log \varepsilon+\partial_{2}+B_{32}\right)\right. \\
& \left.\quad+\partial_{2}\left(\sum_{i, j} x_{i} x_{j} D_{i j}\right)\right]
\end{aligned}
$$

plus terms vanishing at $x_{1}=x_{2}=0$.

Now $J$, and hence $K$, depends on the derivatives of $\varepsilon$ and $\mu$. However, the dependence in $J-J^{\prime}$ comes only from $B_{33}-B_{33}^{\prime}$ for

$$
J-J^{\prime}=\left(\mathrm{Id}+\sum_{i, j} x_{i} x_{j} D_{i j}\right)\left[B_{33}-B_{33}^{\prime}+\partial_{3} \log \left(\frac{\varepsilon}{\varepsilon^{\prime}}\right)\right]
$$

and as we are working at $x_{3}=0, \varepsilon=\varepsilon^{\prime}$. Furthermore, at $x_{3}=0, B_{33}$ and $B_{33}^{\prime}$ can differ by an operator of order at most $-(l-1)$. If two first order operators are equal up to order $-(l-1)$ then using

$$
a^{-1}-b^{-1}=a^{-1}(b-a) b^{-1}
$$

we conclude that their parametrices agree to order $-(l+1)$. So we have that $K^{\prime}-K$ is of order $-(l+1)$, where $K^{\prime}$ is the analogue of $K$ for $\varepsilon^{\prime}, \mu^{\prime}$, and its principal symbol at $p$ will be $-\left|\xi^{\prime}\right|_{x}^{-2} \sigma_{-(l-1)}\left(F_{33}\right)$.

And so

$$
\begin{aligned}
& i \omega \mu\left(\Lambda_{11}^{\prime}-\Lambda_{11}\right)=\left(B_{12}+F_{12}\right) \\
& -\left(B_{13}+F_{13}-\partial_{1}\right) K^{\prime}\left[\left(\operatorname{Id}+\sum_{i, j} x_{i} x_{j} D_{i j}\right)\left(\partial_{2} \log \varepsilon^{\prime}+\partial_{2}+B_{32}+F_{32}\right)\right. \\
& \left.+\partial_{2}\left(\sum_{i, j} x_{i} x_{j} D_{i j}\right)\right]-B_{12} \\
& +\left(B_{13}-\partial_{1}\right) K\left[\left(\operatorname{Id}+\sum_{i, j} x_{i} x_{j} D_{i j}\right)\left(\partial_{2} \log \varepsilon+\partial_{2}+B_{32}\right)\right.
\end{aligned}
$$




$$
\begin{aligned}
& \left.+\partial_{2}\left(\sum_{i, j} x_{i} x_{j} D_{i j}\right)\right] \\
& =F_{12}-\left(B_{13}-\partial_{1}\right) K^{\prime}\left(\mathrm{Id}+\sum_{i, j} x_{i} x_{j} D_{i j}\right) F_{32}-\left(B_{13}-\partial_{1}\right)\left(K^{\prime}-K\right) \\
& \cdot\left[\left(\mathrm{Id}+\sum_{i, j} x_{i} x_{j} D_{i j}\right)\left(\partial_{2}+B_{32}\right)+\partial_{2}\left(\sum_{i, j} x_{i} x_{j} D_{i j}\right)\right] \\
& -F_{13} K^{\prime}\left[\left(\operatorname{Id}+\sum_{i, j} x_{i} x_{j} D_{i j}\right)\left(\partial_{2} \log \varepsilon^{\prime}+\partial_{2}+B_{32}+F_{32}\right)\right. \\
& \left.+\partial_{2}\left(\sum_{i, j} x_{i} x_{j} D_{i j}\right)\right] \\
& -\left(B_{13}-\partial_{1}\right)\left[\left(K^{\prime}-K\right)\left(\mathrm{Id}+\sum_{i, j} x_{i} x_{j} D_{i j}\right) \partial_{2} \log \varepsilon^{\prime}\right. \\
& \left.+K\left(\operatorname{Id}+\sum_{i, j} x_{i} x_{j} D_{i j}\right) \partial_{2} \log \left(\frac{\varepsilon^{\prime}}{\varepsilon}\right)\right] \text {. }
\end{aligned}
$$

The principal symbol of this at $p$ will come from

$$
F_{12}+\partial_{1} K^{\prime} F_{32}+\partial_{1}\left(K^{\prime}-K\right) \partial_{2}-F_{13} K^{\prime}\left(\partial_{2}+F_{32}\right) .
$$

Using the results of the previous section, if $m=-(l-1)$, this has principal symbol at the point $p$ equal to

$$
\begin{gathered}
\sigma_{m}\left(F_{12}\right)-i \xi_{1}\left|\xi^{\prime}\right|_{x}^{-1} \sigma_{m}\left(F_{32}\right)+\xi_{1} \xi_{2}\left|\xi^{\prime}\right|_{x}^{-2} \sigma_{m}\left(F_{33}\right) \\
+\left|\xi^{\prime}\right|_{x}^{-1} \sigma_{m}\left(F_{13}\right)\left(i \xi_{2}+\sigma_{m}\left(F_{32}\right)\right)
\end{gathered}
$$

which by (1.3) is equal to

$$
\frac{l !}{2^{l}\left|\xi^{\prime}\right|_{x}^{l+1}}\left(\xi_{1} \xi_{2} e_{\varepsilon}-\xi_{1} \xi_{2}\left(e_{\varepsilon}+e_{\mu}\right)\right)=\frac{-l !}{2^{l}\left|\xi^{\prime}\right|_{x}^{l+1}} \xi_{1} \xi_{2} e_{\mu}
$$

Computing the remaining components of the principal symbol of $\Lambda^{\prime}-\Lambda$ at $p$, we obtain

$$
0=\frac{-l !}{2^{l}\left|\xi^{\prime}\right|_{x}^{l+1}} \frac{e_{\mu}}{i \omega \mu}\left(\begin{array}{cc}
-\xi_{1} \xi_{2} & -\xi_{2}^{2} \\
\xi_{1}^{2} & \xi_{1} \xi_{2}
\end{array}\right)
$$

Thus we are able to determine $e_{\mu}$ from $\Lambda^{\prime}-\Lambda$. Since we are assuming knowledge of the map $\Lambda$, we know also its inverse; restricted to the space $T H_{\text {Div }}^{\frac{1}{2}}(\partial \Omega)$, this is the impedance map which maps the tangential component 
of the magnetic field at the boundary to that of the electric field at the boundary. Interchanging the roles of $\varepsilon$ and $\mu$ in our analysis, we find that computing the principal symbol of $\left(\Lambda^{\prime}\right)^{-1}-\Lambda^{-1}$ yields

$$
0=\frac{l !}{2^{l}\left|\xi^{\prime}\right|_{x}^{l+1}} \frac{e_{\varepsilon}}{i \omega \varepsilon}\left(\begin{array}{cc}
-\xi_{1} \xi_{2} & -\xi_{2}^{2} \\
\xi_{1}^{2} & \xi_{1} \xi_{2}
\end{array}\right)
$$

and we may conclude that $e_{\varepsilon}$ and $e_{\mu}$ are determined at the boundary from the assumed knowledge of the boundary maps.

We remark the unexpected fact that the inverse of the first order nonelliptic pseudo-differential operator exists, and that it is also a first order pseudo-differential operator; of course, the symbol of the inverse is not the inverse of the symbol. This is a result of working on the restricted $T H_{\text {Div }}^{\frac{1}{2}}(\partial \Omega)$ spaces rather than on Sobolev spaces - see [10] for further discussion.

\section{Chiral Media.}

Suppose now that $\Omega$ is a body with chirality described by a smooth function $\beta$. We shall apply the techniques of the previous section to show that if two bodies have the same admittance map, then the chirality together with the other three material parameters of the bodies must coincide to infinite order at the boundary.

2.1. The equations for chiral media. Working again with one-forms, and taking the formulation of [8] which is a change of variables in the BornFedorov formulation, Maxwell's equations for a chiral body take the form

$$
\begin{aligned}
& * d H=-i \omega(\varepsilon E+\beta H) \\
& * d E=i \omega(\mu H-\beta E)
\end{aligned}
$$

together with

$$
\delta(\varepsilon E+\beta H)=\delta(\mu H-\beta E)=0 .
$$

We write (2.1) as

$$
* d\left(\begin{array}{l}
E \\
H
\end{array}\right)=-i \omega\left(\begin{array}{cc}
\beta & -\mu \\
\varepsilon & \beta
\end{array}\right)\left(\begin{array}{l}
E \\
H
\end{array}\right)=-i \omega X\left(\begin{array}{l}
E \\
H
\end{array}\right)
$$

where we introduce the notation

$$
X=\left(\begin{array}{cc}
\beta & -\mu \\
\varepsilon & \beta
\end{array}\right) .
$$

We have

$$
\begin{aligned}
* d * d\left(\begin{array}{c}
E \\
H
\end{array}\right) & =-i \omega * d\left[X\left(\begin{array}{c}
E \\
H
\end{array}\right)\right] \\
& =-i \omega *\left[d X \wedge\left(\begin{array}{c}
E \\
H
\end{array}\right)\right]-\omega^{2} X^{2}\left(\begin{array}{l}
E \\
H
\end{array}\right)
\end{aligned}
$$


where we have used (2.1) again. But

$$
\left(\begin{array}{l}
E \\
H
\end{array}\right)=-\frac{1}{i \omega} X^{-1} * d\left(\begin{array}{l}
E \\
H
\end{array}\right)
$$

so

$$
* d * d\left(\begin{array}{c}
E \\
H
\end{array}\right)=*\left[d X \wedge X^{-1} * d\left(\begin{array}{c}
E \\
H
\end{array}\right)\right]-\omega^{2} X^{2}\left(\begin{array}{l}
E \\
H
\end{array}\right)
$$

and thus

$$
-\Delta\left(\begin{array}{c}
E \\
H
\end{array}\right)+d * d *\left(\begin{array}{l}
E \\
H
\end{array}\right)-*\left[d X \wedge X^{-1} * d\left(\begin{array}{c}
E \\
H
\end{array}\right)\right]+\omega^{2} X^{2}\left(\begin{array}{l}
E \\
H
\end{array}\right)=0 .
$$

But (2.2) implies that

$$
d * d *\left(\begin{array}{c}
E \\
H
\end{array}\right)=-d\left[X^{-1}(d X) \cdot\left(\begin{array}{c}
E \\
H
\end{array}\right)\right]
$$

where the inner product on one-forms is that induced from the metric, and where the inner product is taken componentwise within the matrix multiplication. Combining these,

$$
\begin{aligned}
-\Delta\left(\begin{array}{l}
E \\
H
\end{array}\right)-d\left[X^{-1}(d X) \cdot\left(\begin{array}{c}
E \\
H
\end{array}\right)\right]-*\left[d X \wedge X^{-1} * d\left(\begin{array}{c}
E \\
H
\end{array}\right)\right] & \\
& +\omega^{2} X^{2}\left(\begin{array}{c}
E \\
H
\end{array}\right)=0
\end{aligned}
$$

which can be written in the form

$$
\mathcal{N}\left(\begin{array}{l}
E \\
H
\end{array}\right)=\left[D_{x_{3}}^{2} I-\Delta_{x_{3}}^{\prime} I-N\left(x, D_{x^{\prime}}\right)-i Q(x) D_{x_{3}}-S(x)\right]\left(\begin{array}{c}
E \\
H
\end{array}\right)=0
$$

where $N$ consists of all terms involving first order differentiation in $x_{1}$ and $x_{2}, Q$ is the coefficient matrix of $\partial / \partial x_{3}$, and $S$ consists of all zero order terms.

As in Proposition 1.1, there is $C\left(x, D_{x^{\prime}}\right) \in \Psi D O^{1}$ such that

$$
\mathcal{N}=\left(D_{x_{3}} I-i Q(x)-i C\right)\left(D_{x_{3}} I+i C\right),
$$

with principal symbol $-\left|\xi^{\prime}\right|_{x}$ times the identity, and with this choice of principal symbol, $C$ is unique modulo smoothing.

2.2. The symbol of $C^{\prime}-C$. Suppose that we have two bodies $(\Omega ; \varepsilon, \mu, \beta)$ and $\left(\Omega ; \varepsilon^{\prime}, \mu^{\prime}, \beta^{\prime}\right)$ for which the admittance maps are identical, and for which the parameters are equal up to order $l$ at the boundary, with $l \geq 2$. As before we shall use' to denote all operators associated with the parameters $\varepsilon^{\prime}, \mu^{\prime}, \beta^{\prime}$. We shall prove that equality of the admittance maps implies that in fact the parameters must agree to order $l+1$, and thus inductively prove that they are equal to infinite order at the boundary. We assume that we have chosen coordinates normal about some point $p$ in the boundary, and 
have extended these normally away from the boundary to a neighborhood of $p$. If we write $C^{\prime}=C+F$ then we have

$$
\begin{gathered}
\Delta^{\prime}+i\left[D_{x_{3}}, C\right]+N+Q C+C^{2}+S=0, \\
\Delta^{\prime}+i\left[D_{x_{3}}, C+F\right]+N^{\prime}+Q^{\prime}(C+F)+(C+F)^{2}+S^{\prime}=0
\end{gathered}
$$

and subtracting yields

$$
i\left[D_{x_{3}}, F\right]+Q^{\prime} F+F^{2}+F C+C F=\left(N^{\prime}-N\right)+\left(S^{\prime}-S\right)+\left(Q^{\prime}-Q\right) C .
$$

The terms on the right hand side of this expression come from

$$
\begin{gathered}
d\left[\left(X^{\prime}\right)^{-1}\left(d X^{\prime}\right) \cdot\left(\begin{array}{c}
E \\
H
\end{array}\right)\right]-d\left[X^{-1}(d X) \cdot\left(\begin{array}{c}
E \\
H
\end{array}\right)\right], \\
*\left[d X^{\prime} \wedge\left(X^{\prime}\right)^{-1} * d\left(\begin{array}{c}
E \\
H
\end{array}\right)\right]-*\left[d X \wedge X^{-1} * d\left(\begin{array}{c}
E \\
H
\end{array}\right)\right], \text { and } \\
\omega^{2} X^{2}\left(\begin{array}{c}
E \\
H
\end{array}\right)-\omega^{2}\left(X^{\prime}\right)^{2}\left(\begin{array}{c}
E \\
H
\end{array}\right) .
\end{gathered}
$$

Specifically, the terms involving differentiation in the tangential variables make up $\left(N^{\prime}-N\right)$, and those involving no differentiation make up $\left(S^{\prime}-\right.$ $S$ ); the coefficients of differentiation with respect to the normal variable $x_{3}$ are what comprise $\left(Q^{\prime}-Q\right)$, which then multiplies the first order pseudodifferential operator $C$. We compute modulo $\Psi D O^{1, l}$ and make use of the following lemma.

Lemma 2.1. (i) If $A$ is a matrix (of 0,1 , or 2 -forms), then terms from (2.4), (2.5) and (2.6) of the form $x_{3}^{l} A \wedge \frac{\partial}{\partial x_{3}}\left(\begin{array}{l}E \\ H\end{array}\right)$ contribute terms in $\Psi D O^{1, l}$ to the right hand side of (2.3).

(ii) If $D$ is a differential operator of order one in $x_{1}$ and $x_{2}$, and of order zero in $x_{3}$, then $x_{3}^{l} D\left(\begin{array}{l}E \\ H\end{array}\right) \in \Psi D O^{1, l}$.

(iii) If $A$ is a matrix of functions, then $x_{3}^{l-1} A\left(\begin{array}{c}E \\ H\end{array}\right) \in \Psi D O^{l-1,0} \subset \Psi D O^{1, l}$.

Proof. Claims (ii) and (iii) are immediate. For (i), we must simply observe that $C$ is a first order pseudo-differential operator in $x_{1}$ and $x_{2}$, and depends smoothly on $x_{3}$ as a parameter. Therefore, in the right hand side of (2.3) we have $x_{3}^{l} A C \in \Psi D O^{1, l}$.

We put

$$
X^{\prime}-X=x_{3}^{l} \mathcal{E}=x_{3}^{l}\left(\begin{array}{cc}
e_{\beta} & -e_{\mu} \\
e_{\varepsilon} & e_{\beta}
\end{array}\right)
$$

and endeavor to show that $\mathcal{E}=0$ at the boundary. 
Proposition 2.2. Suppose $(\varepsilon, \mu, \beta)$ and $\left(\varepsilon^{\prime}, \mu^{\prime}, \beta^{\prime}\right)$ are equal to order $l \geq 2$ at the boundary. Writing $C^{\prime}=C+F$, we have $F \in \Psi D O^{0, l-1}$, and if $\left(f_{j}\right)_{j=0,-(l-1)}$ is the principal symbol of $F$ at the boundary then in our chosen local boundary normal coordinates,

$$
\begin{aligned}
& f_{-(l-1)}=\frac{-l !}{2^{l}\left|\xi^{\prime}\right|_{x}^{l}\left(\varepsilon \mu+\beta^{2}\right)}\left(\begin{array}{ll}
W_{11} & W_{12} \\
W_{21} & W_{22}
\end{array}\right)+r^{\prime} \text {, where } \\
& W_{11}=\left(\begin{array}{ccc}
\left|\xi^{\prime}\right|_{x}\left(\beta e_{\beta}+\varepsilon e_{\mu}\right) & 0 & i \xi_{1}\left(2 \beta e_{\beta}+\varepsilon e_{\mu}+\mu e_{\varepsilon}\right) \\
0 & \left|\xi^{\prime}\right|_{x}\left(\beta e_{\beta}+\varepsilon e_{\mu}\right) & i \xi_{2}\left(2 \beta e_{\beta}+\varepsilon e_{\mu}+\mu e_{\varepsilon}\right) \\
0 & 0 & \left|\xi^{\prime}\right|_{x}\left(\beta e_{\beta}+\mu e_{\varepsilon}\right)
\end{array}\right) \text {, } \\
& W_{12}=\left(\begin{array}{ccc}
\left|\xi^{\prime}\right|_{x}\left(\mu e_{\beta}-\beta e_{\mu}\right) & 0 & i \xi_{1}\left(2 \mu e_{\beta}-2 \beta e_{\mu}\right) \\
0 & \left|\xi^{\prime}\right|_{x}\left(\mu e_{\beta}-\beta e_{\mu}\right) & i \xi_{2}\left(2 \mu e_{\beta}-2 \beta e_{\mu}\right) \\
0 & 0 & \left|\xi^{\prime}\right|_{x}\left(\mu e_{\beta}-\beta e_{\mu}\right)
\end{array}\right), \\
& W_{21}=\left(\begin{array}{ccc}
\left|\xi^{\prime}\right|_{x}\left(\beta e_{\varepsilon}-\varepsilon e_{\beta}\right) & 0 & i \xi_{1}\left(2 \beta e_{\varepsilon}-2 \varepsilon e_{\beta}\right) \\
0 & \left|\xi^{\prime}\right|_{x}\left(\beta e_{\varepsilon}-\varepsilon e_{\beta}\right) & i \xi_{2}\left(2 \beta e_{\varepsilon}-2 \varepsilon e_{\beta}\right) \\
0 & 0 & \left|\xi^{\prime}\right|_{x}\left(\beta e_{\varepsilon}-\varepsilon e_{\beta}\right)
\end{array}\right), \quad \text { and } \\
& W_{22}=\left(\begin{array}{ccc}
\left|\xi^{\prime}\right|_{x}\left(\beta e_{\beta}+\mu e_{\varepsilon}\right) & 0 & i \xi_{1}\left(2 \beta e_{\beta}+\varepsilon e_{\mu}+\mu e_{\varepsilon}\right) \\
0 & \left|\xi^{\prime}\right|_{x}\left(\beta e_{\beta}+\mu e_{\varepsilon}\right) & i \xi_{2}\left(2 \beta e_{\beta}+\varepsilon e_{\mu}+\mu e_{\varepsilon}\right) \\
0 & 0 & \left|\xi^{\prime}\right|_{x}\left(\beta e_{\beta}+\varepsilon e_{\mu}\right)
\end{array}\right)
\end{aligned}
$$

and where $r^{\prime}$ vanishes to second order at $x_{1}, x_{2}=0$.

Proof. We first analyze (2.4):

$$
\begin{aligned}
d & {\left[\left(\left(X^{\prime}\right)^{-1} d X^{\prime}-X^{-1} d X\right) \cdot\left(\begin{array}{c}
E \\
H
\end{array}\right)\right] } \\
= & d\left[\left(X^{-1} d\left(X^{\prime}-X\right)+\left(X^{\prime}\right)^{-1}\left(X-X^{\prime}\right) X^{-1} d X^{\prime}\right) \cdot\left(\begin{array}{c}
E \\
H
\end{array}\right)\right] \\
= & d\left[\left(X^{-1} d\left(x_{3}^{l} \mathcal{E}\right)-x_{3}^{l}\left(X^{\prime}\right)^{-1} \mathcal{E} X^{-1} d X^{\prime}\right) \cdot\left(\begin{array}{c}
E \\
H
\end{array}\right)\right] \\
= & l(l-1) x_{3}^{l-2} X^{-1} \mathcal{E}\left(\begin{array}{c}
E_{3} \\
H_{3}
\end{array}\right) d x_{3}+l x_{3}^{l-1} d\left(X^{-1} \mathcal{E}\right)\left(\begin{array}{c}
E_{3} \\
H_{3}
\end{array}\right) \\
& +l x_{3}^{l-1} X^{-1} \mathcal{E}\left(\begin{array}{c}
d E_{3} \\
d H_{3}
\end{array}\right)+l x_{3}^{l-1} X^{-1} d \mathcal{E} \cdot\left(\begin{array}{c}
E \\
H
\end{array}\right) d x_{3} \\
& +x_{3}^{l} d\left(X^{-1}\right) d \mathcal{E} \cdot\left(\begin{array}{c}
E \\
H
\end{array}\right)+x_{3}^{l} X^{-1} d\left[d \mathcal{E} \cdot\left(\begin{array}{c}
E \\
H
\end{array}\right)\right] \\
& -l x_{3}^{l-1}\left(X^{\prime}\right)^{-1} \mathcal{E} X^{-1} d X^{\prime} \cdot\left(\begin{array}{c}
E \\
H
\end{array}\right) d x_{3}-x_{3}^{l} d\left(\left(X^{\prime}\right)^{-1} \mathcal{E} X^{-1}\right) d X^{\prime} \cdot\left(\begin{array}{c}
E \\
H
\end{array}\right) \\
& -x_{3}^{l}\left(X^{\prime}\right)^{-1} \mathcal{E} X^{-1} d\left[d X^{\prime} \cdot\left(\begin{array}{c}
E \\
H
\end{array}\right)\right]
\end{aligned}
$$




$$
=\sum_{i=1}^{9} T_{i}, \quad \text { say. }
$$

From Lemma 2.1 terms $T_{2}$ and $T_{4}, \ldots, T_{9}$ contribute terms in $\Psi D O^{1, l}$ to the right hand side of (2.3). Writing

$$
T_{3}=l x_{3}^{l-1} X^{-1} \mathcal{E}\left(\begin{array}{c}
\partial_{1} E_{3} d x_{1}+\partial_{2} E_{3} d x_{2} \\
\partial_{1} H_{3} d x_{1}+\partial_{2} H_{3} d x_{2}
\end{array}\right)+l x_{3}^{l-1} X^{-1} \mathcal{E}\left(\begin{array}{c}
\partial_{3} E_{3} d x_{3} \\
\partial_{3} H_{3} d x_{3}
\end{array}\right)
$$

and recalling that the coefficient of the term involving $\partial x_{3}$ multiplies $C$ in the right hand side of (2.3), we have the contribution from (2.4) equal to

$$
l(l-1) x_{3}^{l-2} X^{-1} \mathcal{E}\left(\begin{array}{cc}
Y_{1} & 0 \\
0 & Y_{1}
\end{array}\right)+l x_{3}^{l-1} X^{-1} \mathcal{E}\left(\begin{array}{cc}
Z_{1} & 0 \\
0 & Z_{1}
\end{array}\right)
$$

where

$$
Y_{1}=\left(\begin{array}{ccc}
0 & 0 & 0 \\
0 & 0 & 0 \\
0 & 0 & 1
\end{array}\right), \quad \text { and } \quad Z_{1}=\left(\begin{array}{ccc}
0 & 0 & \partial_{1} \\
0 & 0 & \partial_{2} \\
0 & 0 & C_{33}
\end{array}\right)
$$

with $C_{33}$ the 33-component of $C$. Next,

$$
\begin{aligned}
* & {\left[d X^{\prime} \wedge\left(X^{\prime}\right)^{-1} * d\left(\begin{array}{c}
E \\
H
\end{array}\right)\right]-*\left[d X \wedge X^{-1} * d\left(\begin{array}{c}
E \\
H
\end{array}\right)\right] } \\
= & *\left[d X^{\prime} \wedge\left[\left(X^{\prime}\right)^{-1}\left(X-X^{\prime}\right) X^{-1}+X^{-1}\right] * d\left(\begin{array}{c}
E \\
H
\end{array}\right)\right] \\
& -*\left[d X \wedge X^{-1} * d\left(\begin{array}{c}
E \\
H
\end{array}\right)\right] \\
= & *\left[d X^{\prime} \wedge\left(X^{\prime}\right)^{-1} x_{3}^{l} \mathcal{E} X^{-1} * d\left(\begin{array}{c}
E \\
H
\end{array}\right)\right]+*\left[l x_{3}^{l-1} \mathcal{E} d x_{3} \wedge X^{-1} * d\left(\begin{array}{c}
E \\
H
\end{array}\right)\right] \\
& +*\left[x_{3}^{l} d \mathcal{E} \wedge X^{-1} * d\left(\begin{array}{c}
E \\
H
\end{array}\right)\right] .
\end{aligned}
$$

The first and third of these terms contribute elements of $\Psi D O^{1, l}$ by Lemma 2.1. For the second term we write $*=\left(\operatorname{Id}+x_{3} D+\sum_{i, j \leq 2} x_{i} x_{j} D_{i j}\right) *_{f}$ to obtain

$*\left[l x_{3}^{l-1} \mathcal{E} d x_{3} \wedge X^{-1} * d\left(\begin{array}{c}E \\ H\end{array}\right)\right]=*_{f}\left[l x_{3}^{l-1} \mathcal{E} d x_{3} \wedge X^{-1} *_{f} d\left(\begin{array}{c}E \\ H\end{array}\right)\right]+T+T^{\prime}$

where $T$ results in terms in $\Psi D O^{1, l}$ by Lemma 2.1 again, and $T^{\prime}$ vanishes to second order at $x_{1}, x_{2}=0$. Computing in terms of the flat star operator, the contribution from (2.5) is thus

$$
l x_{3}^{l-1} \mathcal{E} X^{-1}\left(\begin{array}{cc}
Z_{2} & 0 \\
0 & Z_{2}
\end{array}\right)\left(\begin{array}{l}
E \\
H
\end{array}\right)+T^{\prime}, \quad \text { where } Z_{2}=\left(\begin{array}{ccc}
-C_{33} & 0 & \partial_{1} \\
0 & -C_{33} & \partial_{2} \\
0 & 0 & 0
\end{array}\right)
$$


The final consideration is the contribution from (2.6). This is

$$
\begin{aligned}
\omega^{2}\left(X^{2}-\left(X^{\prime}\right)^{2}\right)\left(\begin{array}{l}
E \\
H
\end{array}\right) & =\omega^{2}\left[X\left(X-X^{\prime}\right)-\left(X^{\prime}-X\right) X^{\prime}\right]\left(\begin{array}{c}
E \\
H
\end{array}\right) \\
& =\omega^{2}\left[x_{3}^{l} \mathcal{E} X^{\prime}-x_{3}^{l} X \mathcal{E}\right]\left(\begin{array}{c}
E \\
H
\end{array}\right)
\end{aligned}
$$

which is in $\Psi D O^{1, l}$.

As before $F \in \Psi D O^{0, l-1}$, and we now compute the symbol of $F$ at $p$. Let the vector $\left(f_{j}\right)_{j=0,-(l-1)}$ be the principal symbol of $F$ at $x_{3}=0$. From (2.3), (2.7) and (2.8) we have

$$
-2\left|\xi^{\prime}\right|_{x} f_{0}=l X^{-1} \mathcal{E}\left(\begin{array}{cc}
\sigma\left(Z_{1}\right) & 0 \\
0 & \sigma\left(Z_{1}\right)
\end{array}\right)+l \mathcal{E} X^{-1}\left(\begin{array}{cc}
\sigma\left(Z_{2}\right) & 0 \\
0 & \sigma\left(Z_{2}\right)
\end{array}\right)
$$

and

$$
-2\left|\xi^{\prime}\right|_{x} f_{-1}+(l-1) f_{0}=l(l-1) X^{-1} \mathcal{E}\left(\begin{array}{cc}
\sigma\left(Y_{1}\right) & 0 \\
0 & \sigma\left(Y_{2}\right)
\end{array}\right) .
$$

In general,

$$
\begin{aligned}
f_{-j}= & \frac{-l !}{(l-j-1) ! 2^{j}\left|\xi^{\prime}\right|_{x}^{j}}\left[X^{-1} \mathcal{E}\left(\begin{array}{cc}
\sigma\left(Y_{1}\right) & 0 \\
0 & \sigma\left(Y_{1}\right)
\end{array}\right)\right. \\
& \left.+\frac{X^{-1} \mathcal{E}}{2\left|\xi^{\prime}\right|_{x}}\left(\begin{array}{cc}
\sigma\left(Z_{1}\right) & 0 \\
0 & \sigma\left(Z_{1}\right)
\end{array}\right)+\frac{\mathcal{E} X^{-1}}{2\left|\xi^{\prime}\right|_{x}}\left(\begin{array}{cc}
\sigma\left(Z_{2}\right) & 0 \\
0 & \sigma\left(Z_{2}\right)
\end{array}\right)\right]
\end{aligned}
$$

and in particular, computing $f_{-(l-1)}$ we obtain the expression in the statement of the proposition.

2.3. Proof that $\mathcal{E}=0$. From [7], we know that for a chiral body, the parameters and their first normal derivatives are determined by the admittance map. Suppose that $(\varepsilon, \mu, \beta)$ and $\left(\varepsilon^{\prime}, \mu^{\prime}, \beta^{\prime}\right)$ agree to order $l \geq 2$ at the boundary. We show now that if $\Pi=\Pi^{\prime}$, then the parameters must agree to order $l+1$. We fix $p$ in the boundary and work in our chosen boundary normal coordinates near $p$.

In order to be able to express $\Pi$ in terms of the constructed operator $C$, we shall need to write $E_{3}$ and $H_{3}$ in terms of $E_{1}, E_{2}, H_{1}$ and $H_{2}$. From (2.2) we know that

$$
d X \wedge *\left(\begin{array}{c}
E \\
H
\end{array}\right)+X d *\left(\begin{array}{c}
E \\
H
\end{array}\right)=0 .
$$

Writing $*=\left(\operatorname{Id}+x_{3} D+\sum_{i, j \leq 2} x_{i} x_{j} D_{i j}\right) *_{f}$ and computing at $x_{3}=0$, we have

$$
\left(\mathrm{Id}+\sum_{i, j \leq 2} x_{i} x_{j} D_{i j}\right)
$$




$$
\begin{aligned}
& \cdot\left(\begin{array}{c}
\partial_{1} \beta E_{1}+\partial_{2} \beta E_{2}+\partial_{3} \beta E_{3}-\partial_{1} \mu H_{1}-\partial_{2} \mu H_{2}-\partial_{3} \mu H_{3} \\
\partial_{1} \varepsilon E_{1}+\partial_{2} \varepsilon E_{2}+\partial_{3} \varepsilon E_{3}+\partial_{1} \beta H_{1}+\partial_{2} \beta H_{2}+\partial_{3} \beta H_{3}
\end{array}\right) \\
+ & X\left(\begin{array}{l}
\mathrm{Id}+\sum_{i, j \leq 2} x_{i} x_{j} D_{i j}
\end{array}\right)\left(\begin{array}{c}
\partial_{1} E_{1}+\partial_{2} E_{2}+\partial_{3} E_{3} \\
\partial_{1} H_{1}+\partial_{2} H_{2}+\partial_{3} H_{3}
\end{array}\right)+D X\left(\begin{array}{c}
E_{3} \\
H_{3}
\end{array}\right) \\
+ & X d\left(\sum_{i, j \leq 2} x_{i} x_{j} D_{i j}\right) \wedge *_{f}\left(\begin{array}{c}
E \\
H
\end{array}\right)=0 .
\end{aligned}
$$

Making use of the fact that $\partial /\left.\partial_{3}\right|_{x_{3}=0}=C,(2.9)$ can be written

$$
J\left(\begin{array}{l}
E_{3} \\
H_{3}
\end{array}\right)=\left(\begin{array}{l}
R_{1} \\
R_{2}
\end{array}\right)
$$

where

$$
\begin{aligned}
J= & \left(\operatorname{Id}+\sum_{i, j \leq 2} x_{i} x_{j} D_{i j}\right)\left[\left(\begin{array}{cc}
\partial_{3} \beta & -\partial_{3} \mu \\
\partial_{3} \varepsilon & \partial_{3} \beta
\end{array}\right)+X\left(\begin{array}{ll}
C_{33} & C_{36} \\
C_{63} & C_{66}
\end{array}\right)\right] \\
& +\left(D+\partial_{3}\left(\sum_{i, j \leq 2} x_{i} x_{j} D_{i j}\right)\right) X
\end{aligned}
$$

and

$$
\begin{aligned}
R= & -\left(\operatorname{Id}+\sum_{i, j \leq 2} x_{i} x_{j} D_{i j}\right)\left[\left(\begin{array}{c}
\partial_{1} \beta E_{1}+\partial_{2} \beta E_{2}-\partial_{1} \mu H_{1}-\partial_{2} \mu H_{2} \\
\partial_{1} \varepsilon E_{1}+\partial_{2} \varepsilon E_{2}+\partial_{1} \beta H_{1}+\partial_{2} \beta H_{2}
\end{array}\right)\right. \\
& \left.+X\left(\begin{array}{l}
\left(\partial_{1}+C_{31}\right) E_{1}+\left(\partial_{2}+C_{32}\right) E_{2}+C_{34} H_{1}+C_{35} H_{2} \\
C_{61} E_{1}+C_{62} E_{2}+\left(\partial_{1}+C_{64}\right) H_{1}+\left(\partial_{2}+C_{65}\right) H_{2}
\end{array}\right)\right] \\
& -X\left(\begin{array}{l}
\left(\partial_{1} \sum_{i, j \leq 2} x_{i} x_{j} D_{i j}\right) E_{1}+\left(\partial_{2} \sum_{i, j \leq 2} x_{i} x_{j} D_{i j}\right) E_{2} \\
\left(\partial_{1} \sum_{i, j \leq 2} x_{i} x_{j} D_{i j}\right) H_{1}+\left(\partial_{2} \sum_{i, j \leq 2} x_{i} x_{j} D_{i j}\right) H_{2}
\end{array}\right) .
\end{aligned}
$$

We similarly have expressions for $J^{\prime}$ and $R^{\prime}$.

We now consider the admittance map $\Pi$. In fact we are considering the admittance map together with its inverse (when restricted to $T H_{\text {Div }}^{\frac{1}{2}}(\partial \Omega)$ ) resulting in the $4 \times 4$ system below which we shall continue to refer to as $\Pi$. 
By (2.1) and (1.5) this map is

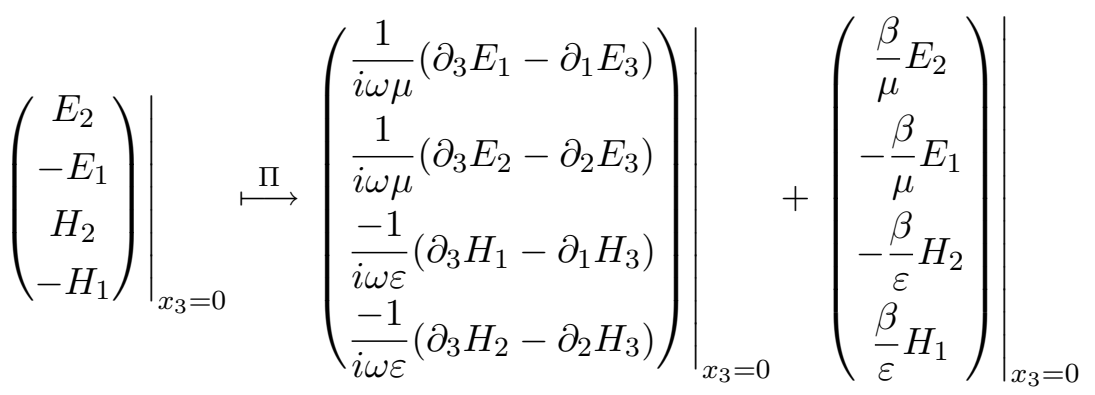

plus terms with coefficients vanishing at $x_{1}=x_{2}=0$; since we shall be computing the symbol of $\Pi^{\prime}-\Pi$, we do not need to consider such terms by Lemma 1.6.

Let $K$ be such that $K J=$ Id modulo smoothing (and similarly define $\left.K^{\prime}\right)$. Note that the principal symbols of $K$ and $K^{\prime}$ at $p$ are both equal to $-\left|\xi^{\prime}\right|_{x}^{-1} X^{-1}$ since $X=X^{\prime}$ and $X^{-1}=\left(X^{\prime}\right)^{-1}$ there. Then

$$
\begin{aligned}
\partial_{3} E_{1}-\partial_{1} E_{3}+i \omega \beta E_{2}= & C_{11} E_{1}+C_{12} E_{2}+C_{14} H_{1}+C_{15} H_{2} \\
& +\left(C_{13}-\partial_{1}\right)(K R)_{1}+C_{16}(K R)_{2}+i \omega \beta E_{2}
\end{aligned}
$$

and similarly, in terms of the second body,

$$
\begin{aligned}
& \partial_{3} E_{1}-\partial_{1} E_{3}+i \omega \beta E_{2} \\
& =\left(C_{11}+F_{11}\right) E_{1}+\left(C_{12}+F_{12}\right) E_{2} \\
& \quad+\left(C_{14}+F_{14}\right) H_{1}+\left(C_{15}+F_{15}\right) H_{2} \\
& \quad+\left(C_{13}+F_{13}-\partial_{1}\right)\left(K^{\prime} R^{\prime}\right)_{1}+\left(C_{16}+F_{16}\right)\left(K^{\prime} R^{\prime}\right)_{2}+i \omega \beta E_{2} .
\end{aligned}
$$

Here $(K R)_{j}$ is the $j^{\text {th }}$ component of the vector $(K R)$, and similarly for $\left(K^{\prime} R^{\prime}\right)_{j}$. Looking at the difference of these two, the first component of the image of $\left(E_{2},-E_{1}, H_{2},-H_{1}\right)^{\prime}$ under $\Pi^{\prime}-\Pi$ (times $\left.i \omega \mu\right)$ is

$$
\begin{aligned}
F_{11} & E_{1}+F_{12} E_{2}+F_{14} H_{1}+F_{15} H_{2}+\left(C_{13}+F_{13}-\partial_{1}\right)\left(K^{\prime} R^{\prime}\right)_{1} \\
& -\left(C_{13}-\partial_{1}\right)(K R)_{1}+\left(C_{16}+F_{16}\right)\left(K^{\prime} R^{\prime}\right)_{2}-C_{16}(K R)_{2} \\
= & F_{11} E_{1}+F_{12} E_{2}+F_{14} H_{1}+F_{15} H_{2}+F_{13}\left(K^{\prime} R^{\prime}\right)_{1}+F_{16}\left(K^{\prime} R^{\prime}\right)_{2} \\
& +C_{13}\left(K^{\prime} R^{\prime}-K R\right)_{1}+C_{16}\left(K^{\prime} R^{\prime}-K R\right)_{2} \\
& +\partial_{1}\left[\left(K-K^{\prime}\right) R\right]_{1}-\partial_{1}\left[K^{\prime}\left(R^{\prime}-R\right)\right]_{1} .
\end{aligned}
$$

Now modulo smoothing,

$$
K-K^{\prime}=K\left(J^{\prime}-J\right) K^{\prime}=K\left(\mathrm{Id}+\sum_{i, j \leq 2} x_{i} x_{j} D_{i j}\right) X^{\prime}\left(\begin{array}{ll}
F_{33} & F_{36} \\
F_{63} & F_{66}
\end{array}\right) K^{\prime}
$$

(recall that we compute at $\left.x_{3}=0\right)$. Thus if $m=-(l-1)$, acting on the vector $\left(E_{2},-E_{1}, H_{2},-H_{1}\right)^{\prime}$, the principal symbol of $\left(K-K^{\prime}\right) R$ at the point 
$p$ is

$$
\sigma_{m-1}\left(\left(K-K^{\prime}\right) R\right)=\frac{-1}{\left|\xi^{\prime}\right|_{x}^{2}}\left(\begin{array}{ll}
\sigma_{m}\left(F_{33}\right) & \sigma_{m}\left(F_{36}\right) \\
\sigma_{m}\left(F_{63}\right) & \sigma_{m}\left(F_{66}\right)
\end{array}\right)\left(\begin{array}{cccc}
i \xi_{2} & -i \xi_{1} & 0 & 0 \\
0 & 0 & i \xi_{2} & -i \xi_{1}
\end{array}\right) .
$$

Next,

$$
R^{\prime}-R=-X\left(\begin{array}{l}
F_{31} E_{1}+F_{32} E_{2}+F_{34} H_{1}+F_{35} H_{2} \\
F_{61} E_{1}+F_{62} E_{2}+F_{64} H_{1}+F_{65} H_{2}
\end{array}\right)
$$

which has symbol of order $m$ equal to zero by Proposition 2.2, and

$$
\sigma_{0}\left(K^{\prime} R^{\prime}\right)=\frac{1}{\left|\xi^{\prime}\right|_{x}}\left(\begin{array}{cccc}
i \xi_{2} & -i \xi_{1} & 0 & 0 \\
0 & 0 & i \xi_{2} & -i \xi_{1}
\end{array}\right) \text {. }
$$

We now put all these results together. Recall that the principal symbol of $C$ is $-\left|\xi^{\prime}\right|_{x}$ times the identity matrix, and we have the expression for $f_{m}$ in Proposition 2.2. The first row of $i \omega \mu\left(\Pi^{\prime}-\Pi\right)$ thus has principal symbol at $p$

$$
\begin{aligned}
& \left(\frac{i \xi_{2}}{\left|\xi^{\prime}\right|_{x}} \sigma_{m}\left(F_{13}\right)+\frac{\xi_{1} \xi_{2}}{\left|\xi^{\prime}\right|_{x}^{2}} \sigma_{m}\left(F_{33}\right),-\sigma_{m}\left(F_{11}\right)-\frac{i \xi_{1}}{\left|\xi^{\prime}\right|_{x}} \sigma_{m}\left(F_{13}\right)-\frac{\xi_{1}^{2}}{\left|\xi^{\prime}\right|_{x}^{2}} \sigma_{m}\left(F_{33}\right),\right. \\
& \left.\frac{i \xi_{2}}{\left|\xi^{\prime}\right|_{x}} \sigma_{m}\left(F_{16}\right)+\frac{\xi_{1} \xi_{2}}{\left|\xi^{\prime}\right|_{x}^{2}} \sigma_{m}\left(F_{36}\right), \quad-\sigma_{m}\left(F_{14}\right)-\frac{i \xi_{1}}{\left|\xi^{\prime}\right|_{x}} \sigma_{m}\left(F_{16}\right)-\frac{\xi_{1}^{2}}{\left|\xi^{\prime}\right|_{x}^{2}} \sigma_{m}\left(F_{36}\right)\right) \\
& =\frac{-l !}{2^{l}\left|\xi^{\prime}\right|_{x}^{l+1}\left(\varepsilon \mu+\beta^{2}\right)}\left(-\xi_{1} \xi_{2}\left(\beta e_{\beta}+\varepsilon e_{\mu}\right), \quad-\xi_{2}^{2}\left(\beta e_{\beta}+\varepsilon e_{\mu}\right),\right. \\
& \left.\quad-\xi_{1} \xi_{2}\left(\mu e_{\beta}-\beta e_{\mu}\right), \quad-\xi_{2}^{2}\left(\mu e_{\beta}-\beta e_{\mu}\right)\right) .
\end{aligned}
$$

Computing the principal symbol at $p$ of the remaining rows of $\Pi^{\prime}-\Pi=0$ and using Proposition 2.2, we find that in fact

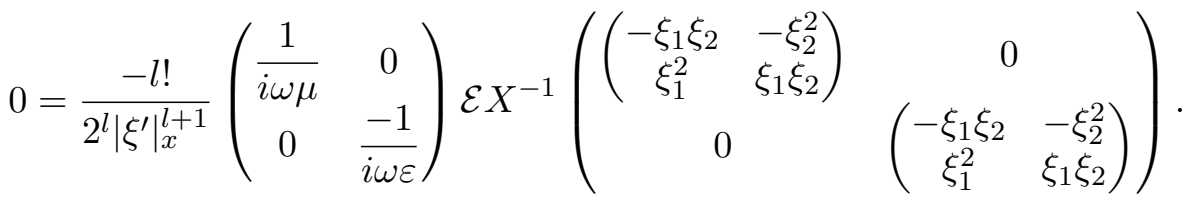

Evaluating, for example, at $\xi_{1}=1, \xi_{2}=0$, we obtain

$$
\left(\begin{array}{cc}
\frac{1}{\mu} & 0 \\
0 & -\frac{1}{\varepsilon}
\end{array}\right) \mathcal{E} X^{-1}=0
$$

and so $\mathcal{E}=0$ as desired.

We remark that one could also argue that the values of $e_{\beta}, e_{\mu}$ are obtainable from the first row and it therefore follows by symmetry that the value of $e_{\epsilon}$ is deducible from the third row and hence that all three values are determined. 


\section{References}

[1] M.S. Joshi, Recovering the total singularity of a potential from backscattering data, preprint.

[2] _ Recovering asymptotics of Coulomb-like potentials, preprint.

[3] M.S. Joshi and A. Sa Barreto, Recovering asymptotics of short range potentials, to appear in Commun on Math. Phys.

[4] - Recovering asymptotics of metrics from fixed energy scattering data, preprint.

[5] A. Lakhtakia, V.K. Varadan and V.V. Varadan, 1989, Time-Harmonic Electromagnetic Fields in Chiral Media, Lecture Notes in Physics, 335 (1989), Berlin, SpringerVerlag.

[6] J. Lee and G. Uhlmann, Determining anisotropic real-analytic conductivities by boundary measurements, Comm. Pure Appl. Math., 42 (1989), 1097-1112.

[7] S. McDowall, 1997, Boundary determination of material parameters from electromagnetic boundary information, Inverse Problems, 13 (1997), 153-163.

[8] _ An electrodynamic inverse problem in chiral media, Trans. AMS, 1997, to appear.

[9] P. Ola, L. Päivärinta and E. Somersalo, An inverse boundary problem in electrodynamics, Duke Math. J. 70 (1993), 617-653.

[10] E. Somersalo, Layer stripping for time harmonic Maxwell's equations with high frequency, Inverse Problems, 10(2) (1994), 449-466.

[11] E. Somersalo, D. Isaacson and M. Cheney, 1992, A linearized inverse boundary value problem for Maxwell's equations, J. Comput. Appl. Math., 42 (1992), 123-136.

Received July 14, 1998. The authors would like to thank the Fields Institute, Toronto, where the work for this paper was initiated.

UNIVERSity OF CAMBRIDGE

CAmbridge CB2 1SB

ENGLAND

E-mail address: joshi@dpmms.cam.ac.uk

UNIVERSITY OF ROCHESTER

ROCHESTER, NY 14627

E-mail address: mcdowall@math.rochester.edu 